\title{
REORIENTATIONAL NONLINEAR PHENOMENA IN THIN NEMATIC LIQUID CRYSTAL LAYER
}

\author{
K. Brzdatiewicz*, W.K. Bajdecki, A. Kozanecka and M.A. Karpierz \\ Faculty of Physics, Warsaw University of Technology \\ Koszykowa 75, 00-662 Warsaw, Poland
}

\begin{abstract}
In this paper the detailed study of the self-diffraction phenomena due to reorientational optical nonlinearity in nematic liquid crystalline cell is presented. The optical nonlinear effect is additionally modified by external low-frequency electric field. The dependence of nonlinear response on light polarization is also analyzed. The theoretical investigations are compared with experimental results and the theoretical predictions are in excellent agreement with experimental data.
\end{abstract}

PACS numbers: 42.65.-k, 42.70.Df

\section{Introduction}

Liquid crystals have plenty of unique physical, optical, electro-, and magnetooptical properties. Therefore, they are very important anisotropic materials in modern optoelectronics. New liquid crystalline mixtures are still being elaborated and their behavior due to external electric or magnetic fields is intensively examined in laboratories. The arrangement occurring in liquid crystals is caused by long-distance interaction between molecules. Their initial orientation is induced by the conditions on boundaries of the liquid crystal cell but even weak external electric, magnetic, or optical field can change this order.

The aim of this work is to study and discuss reorientational phenomena in the thin nematic liquid crystal layer illuminated by a high power laser beam. The influence of the light polarization, texture of liquid crystalline cell, and external low frequency electric field is analyzed both experimentally and theoretically.

Liquid crystals are composed of highly anisotropic and strongly correlated molecules that can be reoriented even by very low external fields. Deformation in nematic liquid crystal causes a change of the free energy. The density of elastic free energy is given by the following expression $[1,2]$ :

$$
f_{\mathrm{F}}=\frac{1}{2} K_{11}(\operatorname{div} n)^{2}+\frac{1}{2} K_{22}(n \cdot \operatorname{rot} \boldsymbol{n})^{2}+\frac{1}{2} K_{33}(n \times \operatorname{rot} \boldsymbol{n})^{2},
$$

*corresponding author; e-mail: kasia@if.pw.edu.pl 
where $n$ is the director (a unit vector defining the mean molecular alignment in the liquid crystal cell), $K_{i i}$ are the Frank (elastic) constants corresponding to three basic types of deformation: splay $(i=1)$, twist $(i=2)$ and bend $(i=3)$.

In our considerations we investigate the liquid crystal cells situated in optical and/or low frequency electric fields. Free energy densities describing influence of those external fields are defined by the formulas

$$
f_{\mathrm{opt}}=-\frac{\varepsilon_{0} \Delta \varepsilon}{4}(\boldsymbol{n} \cdot \boldsymbol{E})^{2}, \quad f_{\mathrm{el}}=-\frac{\varepsilon_{0} \Delta \varepsilon_{\mathrm{st}}}{2}\left(\boldsymbol{n} \cdot \boldsymbol{E}_{\mathrm{st}}\right)^{2},
$$

where $\varepsilon_{0}$ is the permittivity of free space, and $\Delta \varepsilon$ is the electric anisotropy.

Orientation of the liquid crystalline molecules can be calculated from the differential Euler-Lagrange equation, which specifies the minimization of the total free energy of the system and consists of the terms associated with the elastic, optical, magnetic, and electric forces.
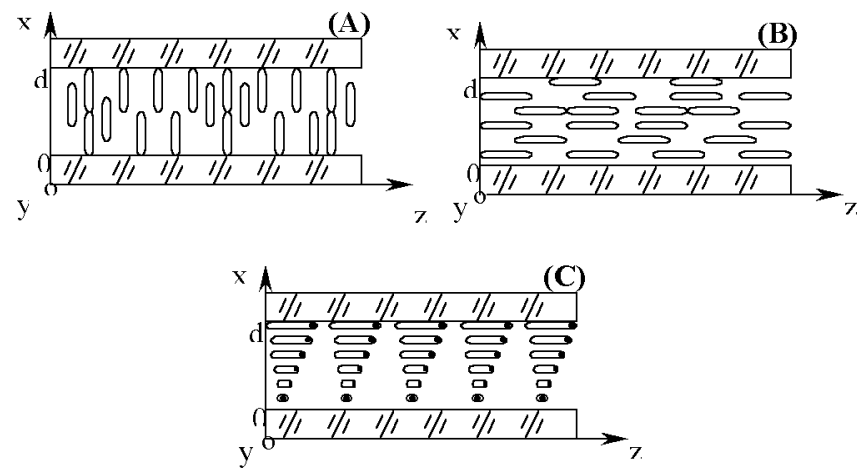

Fig. 1. Schematic drawing of three analyzed types of nematic liquid crystal cell: (A) homeotropic NLC texture, (B) planar NLC texture, (C) twisted NLC texture.

In this paper, three possible textures of nematic liquid crystal (NLC) planar, homeotropic, and twist (all of them are shown in Fig. 1) are analyzed. In calculations we assume configurations, in which the liquid crystalline molecules are oriented in $x z$ plane. Then, the molecular arrangement inside a cell can be strictly described only by one parameter - the orientation angle $\theta$ and the director is simply defined as $\boldsymbol{n}=(\cos \theta, 0, \sin \theta)$. We regard the stationary case, in which reorientation angle does not depend on time. Additionally, the strong anchoring conditions at the boundaries are taken into consideration. It means that molecules are rigidly anchored at boundary surfaces and never change their arrangement. For all numerical calculations realized in our theoretical expectations, we took parameters, which are very close to 4 -trans- $4^{\prime}-n$-hexyl-cyclohexyl-isothiocyanatobenzene (6CHBT) nematic liquid crystal.

\section{Self-diffraction phenomena}

Let us consider homeotropic liquid crystal cell (as seen in Fig. 1A) characterized by the identical conditions on both boundary surfaces: $\theta(x=0)=$ 
$\theta(x=d)=0$. Electromagnetic wave tends to change the homogeneous orientation of the liquid crystal inside the cell. In case of an infinite plane wave with electric field amplitude $E_{z}$, molecular orientation is calculated from the Euler-Lagrange equation in the form

$$
\begin{aligned}
& \frac{\mathrm{d}^{2} \theta}{\mathrm{d} x^{2}}\left(K_{33} \cos ^{2} \theta+K_{11} \sin ^{2} \theta\right)-\frac{1}{2}\left(\frac{\mathrm{d} \theta}{\mathrm{d} x}\right)^{2}\left(K_{33}-K_{11}\right) \sin 2 \theta \\
& +\frac{\varepsilon_{0} \Delta \varepsilon}{4}\left|E_{z}\right|^{2} \sin 2 \theta=0 .
\end{aligned}
$$

For small angles (i.e. $\sin \theta \approx \theta$ ) and for one-elastic-constant approximation (which assigns a single elastic constant $K_{11}=K_{33}=K$ ) it is easy to find the distribution of the orientation angle $\theta$ as a function of an oblong coordinate $x$ : $\theta(x) \sim \sin (\pi x / d)$. Considering the Gaussian character of illuminating beam, i.e. $E=E_{\text {opt }} \exp \left(-r^{2} / w^{2}\right)$ (where $w$ is a beam waist and $r$ is a radius $r^{2}=y^{2}+z^{2}$ ) and neglecting the changes of the beam width during its passing over the cell, the orientation angle can be assumed as $\theta(r, x)=R(r) \sin (\pi x / d)$. In this case differential equation for the orientation transverse profile $R(r)$ takes the form

$$
\begin{aligned}
& \frac{1}{4}\left(K_{11}-K_{33}\right)\left[\left(3 R-\frac{5}{3} R^{3}\right)\left(R_{r}^{2}-2 R_{r}\right)-\left(3 R^{2}-\frac{5}{6} R^{4}\right)\left(R_{r r}+\frac{1}{r} R_{r}\right)\right] \\
& +\frac{1}{4}\left(K_{11}-K_{33}\right) \frac{\pi^{2}}{d^{2}}\left(R^{5}-4 R^{3}\right)-\left(K_{11}+K_{22}\right)\left(R_{r r}+\frac{1}{r} R_{r}\right) \\
& +\frac{2 \pi^{2}}{d^{2}} R K_{33}-2 v E^{2}\left(2 R-R^{3}\right)=0
\end{aligned}
$$

where $R_{r}$ and $R_{r r}$ are the derivatives of $R: R_{r}=\mathrm{d} R / \mathrm{d} r, R_{r r}=\mathrm{d}^{2} R / \mathrm{d} r^{2}$, and $v=\varepsilon_{0} \Delta \varepsilon / 4$. In the one-elastic-constant approximation, Eq. (4) can be simplified to the following expression:

$$
\frac{\mathrm{d}^{2} R}{\mathrm{~d} r^{2}}+\frac{1}{r} \frac{\mathrm{d} R}{\mathrm{~d} r}+2 R\left(\alpha-\alpha_{\mathrm{th}}\right)-R^{3} \alpha=0,
$$

where $\alpha=\alpha_{\text {opt }}=v\left(E_{\text {opt }}\right)^{2} \exp \left(-2 r^{2} / w^{2}\right) / K$, and $\alpha_{\text {th }}=\pi^{2} / 2 d^{2}$ defines the threshold field in an infinite plane wave case.

For a radially symmetric beam incident normally on the liquid crystal cell the reorientation process is the function of the beam radius (in particular, the largest reorientation effect exhibits in the center of the beam). Thus, we have to make allowances for the transverse dependence of molecular reorientation and also for the associated with it nonlinear phase shift. Due to the heterogeneous light intensity distribution, inside the liquid crystal cell the phase diaphragm is created. In consequence, an input Gaussian beam causes the appearance of the diffraction rings as a result of the self-diffraction process [3, 4].

To show the light-induced reorientation nonlinear effects occurring in liquid crystals we found the reorientational angle obtained by a numerical solution of Eq. (5). Then, we calculated the far field diffraction pattern with the aid of the Fourier transform technique. Taking the Fresnel formula, we computed the intensity distribution in the diffraction pattern. Results are plotted in Fig. 2, where 


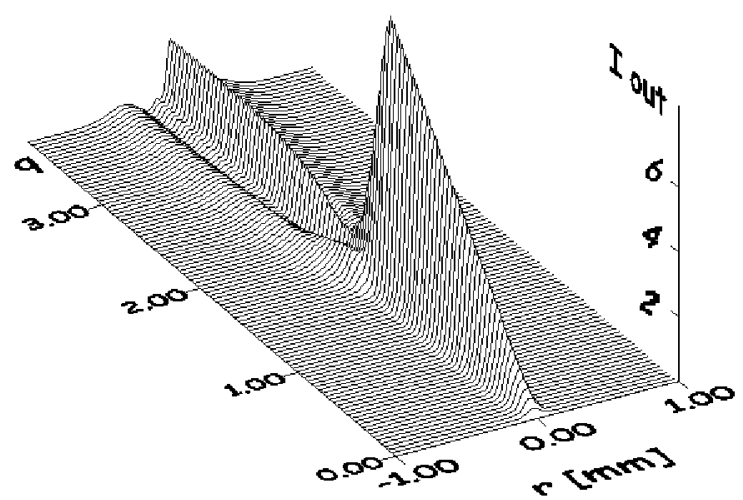

Fig. 2. Diffraction pattern found theoretically for the light intensity $I_{\text {out }}$ (in arbitrary units) in far field screen surface versus the distance $r$ from the center of pattern and the dimensionless parameter $q$ proportional to the input power.

vertical axis is an intensity in the diffraction image, and horizontal axes are respectively: the distance $r$ from the center of picture, and parameter $q$, which is proportional to the input light power and defined as: $q=\alpha_{\mathrm{opt}}(r=0) / \alpha_{\mathrm{th}}$.

The first dark ring appears when the phase difference between the electric fields at the center and at the edge of the beam raises the value of $2 \pi[5]$. Generally, as the result of increasing power of Gaussian beam we obtain the diffraction pattern that consists of more and more thickly arranged concentric rings $[6,7]$.

The relation between the intensity in the diffraction screen surface and the intensities of input signal is sketched in Fig. 3, where we assumed three characteristic points: the middle of the image, the place of first dark ring appearing, and the considerable distance from the center of a diffraction pattern. It is important that the first local maxima of mentioned characteristics are close to each other and they define the approximate value of the light power when nonlinear processes begin. As we can conclude from Fig. 3, the input light power corresponding to parameter $q=1.5$ marks the threshold above which the nonlinear process of molecular reorientation starts. This threshold phenomenon is well known as the Frederiks effect, which generally occurs in nematics reoriented by the perpendicular fields. In addition, we also found that the first dark ring appears for the input light power corresponding to the value $q=2.2$.

Thus, appearing of the reorientation (the Frederiks threshold) for the finite beam is greater than in the infinite planar wave case, and additionally it is close to the value of light power needed to obtain the first diffraction ring.

The experiment was performed with planar aligned 6CHBT liquid crystal cell of $d=10 \mu \mathrm{m}$ thickness. The schematic drawing of the experimental setup is shown in Fig. 4. As a light source an argon laser with the wavelength $\lambda=514 \mathrm{~nm}$ and the spot size of $2 \mathrm{~mm}$ diameter was chosen. The laser beam was focused by the lens $f=40 \mathrm{~mm}$ and from theoretical estimations the light was focused on a liquid crystal cell to the spot size of $13 \mu \mathrm{m}$ diameter.

The optical power meter measured the power of an output signal and the diffraction images were recorded and processed with CCD camera connected to 


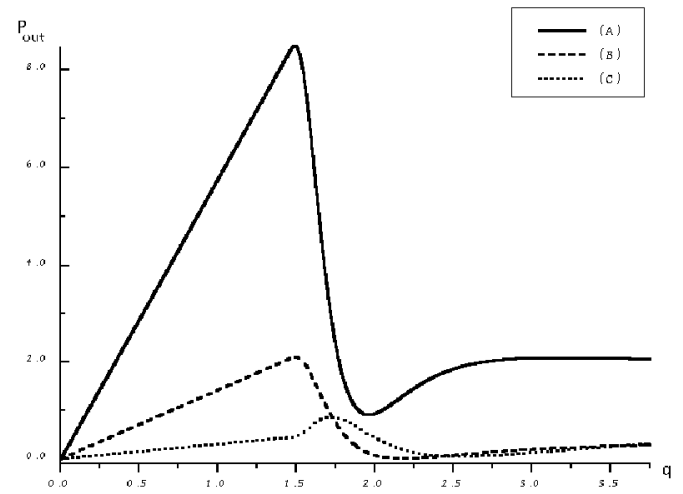

Fig. 3. Output power (in arbitrary units) as a function of parameter $q$ for three points of the diffraction image: $A$ - center of the diffraction pattern, $B$ - place of the first dark diffraction ring appearing, $C$ - region situated in far distance from the center.

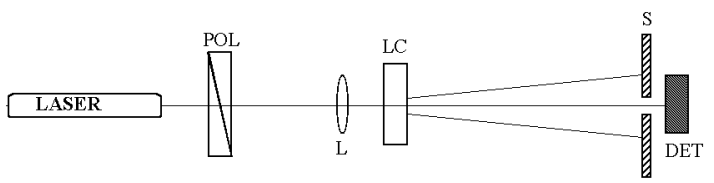

Fig. 4. Experimental setup: POL - polarizer, L - lens, LC - liquid crystal cell, $\mathrm{S}$ - screen, DET - detector.

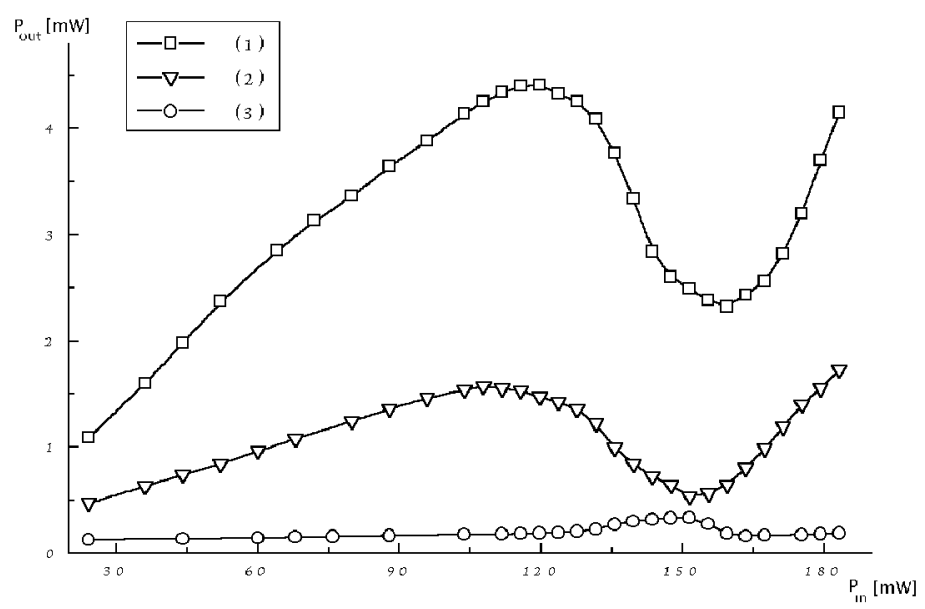

Fig. 5. Output power vs. input power of light for three different detector locations: 1 - at the center of diffraction pattern; 2 - in area of first diffraction ring appearing, 3 - far away from the central point of image.

PC computer. Figure 5 presents the measured relation between the output and input light power for three locations of the detector corresponding to theoretical curves from Fig. 3. 
As was mentioned earlier, for perpendicular incidence of the suitably polarized light wave at the planar aligned nematic, there is no reorientation below a certain value of laser power. From characteristics shown in Fig. 5 we could approximately qualify the threshold of light induced Frederiks transition. Finally, we acknowledged the input power of $110 \mathrm{~mW}$ as a proper one, which is very close to theoretically calculated value, that is equivalent to parameter $q \approx 1.5$ (for experimental data $q=1$ is equivalent to power $P \approx 80 \mathrm{~mW}$ ). An experimental value of input power, which corresponds to the first dark diffraction ring appearing, is about $200 \mathrm{~mW}$ (according to the theoretical value approaches $P \approx 180 \mathrm{~mW}$ ).

\section{Influence of the electric field}

In addition to the optically induced reorientation, an effect of low frequency electric field can be examined, whose influence is in competition or conjunction with the action of optical field.

In this paper we consider the case of opposing influences of both - electric and optical fields. We assume that the low frequency (or static) electric field $E_{\text {st }}$ applied to the NLC layer is normal to the initial molecular orientation and additionally is perpendicular to the electric field of the electromagnetic wave.

Let us consider a planar aligned cell as shown in Fig. 1B. The electric field of the linearly polarized light wave is oscillating in $z$ direction. An electric field (in our experiment we used rectangular signal of $100 \mathrm{~Hz}$ frequency) characterized by its intensity $E_{x}$ is applied to electrodes of the cell.

Both fields rotate molecules to fulfill the conditions of the free energy minimization. In this case we can also take full advantage of Eq. (5), substituting now that $\alpha=\alpha_{\text {st }}-\alpha_{\text {opt }}=\left[v_{\text {st }}\left(E_{z}\right)^{2}-v\left(E_{\text {opt }}\right)^{2} \exp \left(-2 r^{2} / w^{2}\right)\right] / K$.

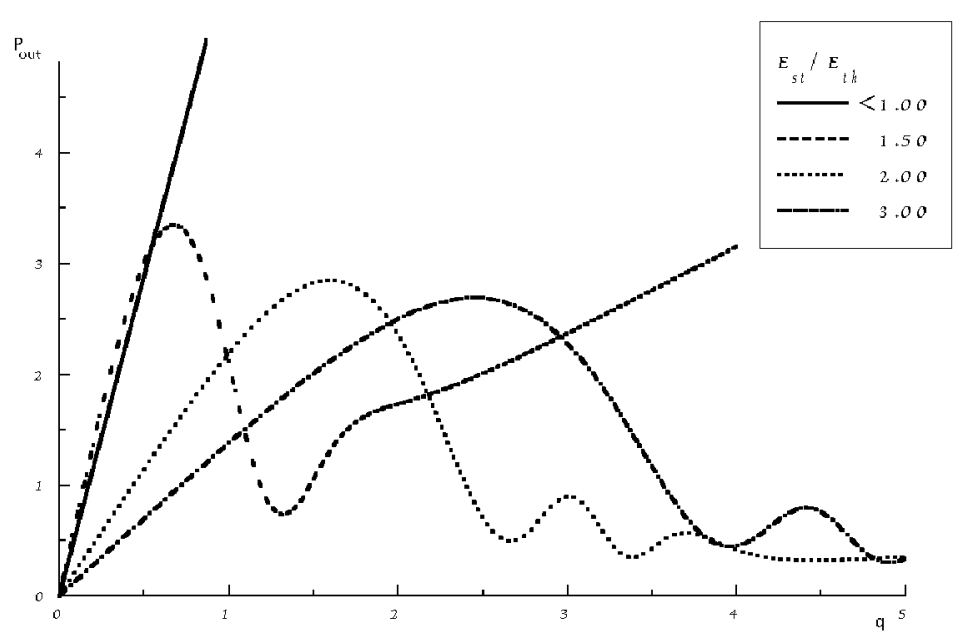

Fig. 6. Theoretical dependence of output power (in arbitrary units) on parameter $q$ for selected values of the electric field $E_{\text {st }}$ (where $E_{\mathrm{th}}$ is a threshold value). 


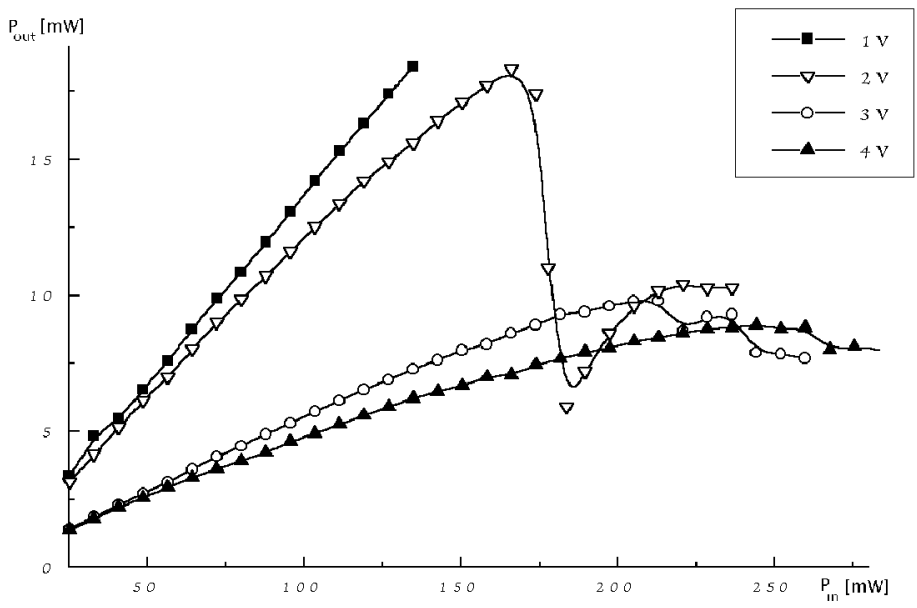

Fig. 7. Variations of the output power measured in the middle of laser beam for increasing power of input light signal and for various voltages of an external electric field.

Theoretically evaluated relation between input and output light power, obtained for different values of additional external electric field intensity are presented in Fig. 6. The conformed experimental results are illustrated by Fig. 7.

In case when the electric field is lower than threshold value $E_{\mathrm{st}}<E_{\mathrm{th}}$, the output light intensity is given as the linear function of the input light power. The increase in the static electric field above the threshold value causes the obtaining of considerable initial reorientation angle. Then, the Gaussian beam with sufficient power can cause so much changes in the arrangement of molecules large enough to induce phase difference necessary for the self-diffraction effects.

Theoretical data obtained by numerical integration of Eq. (5) are in good agreement with the experimental results shown in Fig. 7. Along with an absence of external static electric field and for small value of its intensity (below the threshold which for the examined sample was theoretically estimated at $U_{\text {th }} \approx 1 \mathrm{~V}$ ), we obtained linear dependence between input and output light power. For larger values of applied voltage $\left(>U_{\mathrm{th}}\right.$ ) the first local maximum of characteristics (qualifying the threshold of the self-diffraction process) occurs for higher value of illuminating beam power. For values of $2 \mathrm{~V}, 3 \mathrm{~V}, 4 \mathrm{~V}$ it is respectively: $165 \mathrm{~mW}, 200 \mathrm{~mW}$, and $250 \mathrm{~mW}$ (theoretically $130 \mathrm{~mW}, 190 \mathrm{~mW}$, and $265 \mathrm{~mW}$ ).

\section{Influence of the light polarization}

The nonlinear reorientational effect in nematics is strongly dependent on light polarization. When the light polarization is linear and parallel to the director of liquid crystalline molecules, any reorientational effect does not appear. But in case when the light polarization is not parallel to the director, the process of molecular reorientation occurs generally. However, due to the high anisotropy of liquid crystals the state of light polarization is being changed in the sample. Consequently, the modifications of orientation in $\mathrm{LC}$ cell are dependent upon the 
initial light polarization. This phenomenon is especially observed in twisted nematic texture. The boundary conditions for twist configuration (see Fig. 1C) are not the same on both surfaces: $\theta(x=0)=\pi / 2$ and $\theta(x=d)=0$. Therefore, the polarization of light should suffer a change inside the cell for any input light polarizations. Theoretically obtained dependence of reorientation on the input light polarization is presented in Fig. 8. It corroborates that for two different input light polarizations, changes of the orientation inside the cell are dramatically different and then nonlinear responses are also disparate.
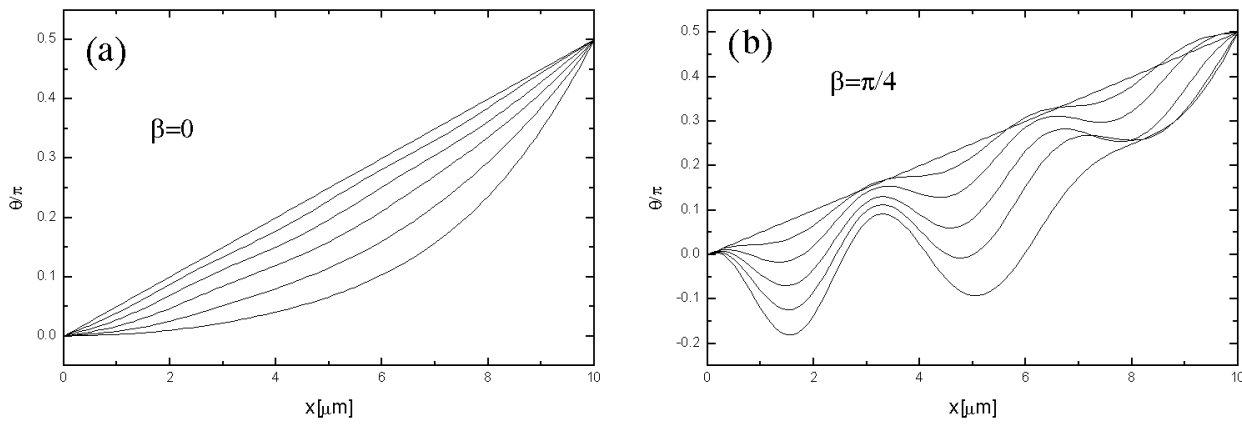

Fig. 8. Orientation angle $\theta$ distribution in twisted nematic liquid crystalline cell for increasing power of light illuminating the sample when the input light linear polarization (a) is parallel to liquid crystalline molecules at the boundary, (b) is rotated by angle $\beta=\pi / 4$.

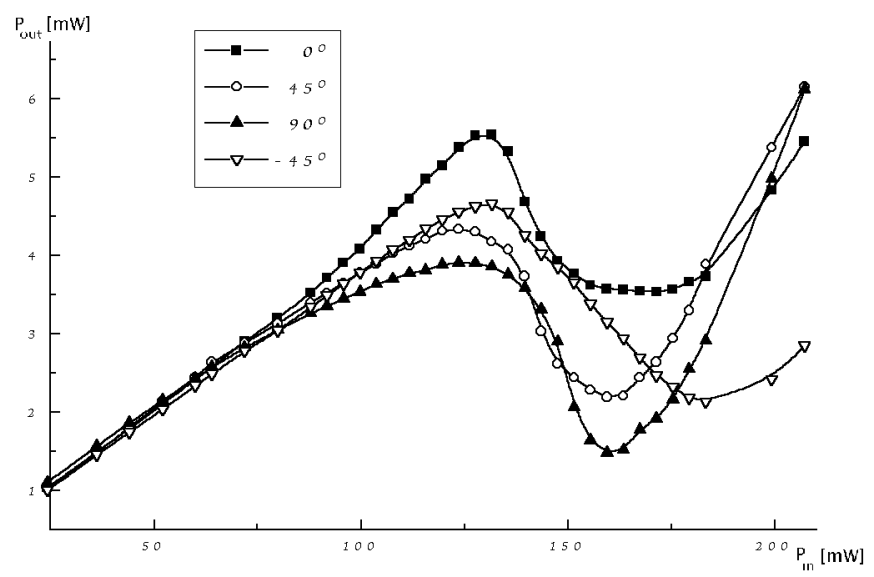

Fig. 9. Input-output characteristics obtained for different polarizations of the light wave illuminating the twisted nematic LC cell.

In our examination we used 6CHBT - twisted nematic cell of $10 \mu \mathrm{m}$ thickness. After placing it in an experimental setup (from Fig. 4) and repeating measurements like in case of planar texture, we received the input-output power characteristics similar to those shown in Fig. 5. We also investigated experimentally the 
influence of the light polarization upon reorientation effects - received results are presented in Fig. 9. Note that reorientation starts at lower power than in samples with threshold.

\section{Conclusions}

The reorientational nonlinearity has a lot of properties, which are unattainable in cases of other types of optical nonlinearity. In particular, it is characterized by strong dependence on the system geometry and light polarization. The different orientations of liquid crystal molecules are corresponding to different nonlinear properties. For example, choosing an initial orientation in the NLC cell we can obtain threshold character of nonlinear processes. Secondly, in consequence of strong dependence of reorientational nonlinearity upon light polarization - some nonlinear effects can appear for one polarization and disappear for the other one.

All our considerations were conducted for reorientational nonlinearity and it was assumed that the result of a thermal nonlinearity influences only quantitatively but not qualitatively on observed effects.

The theoretical predictions and the experimental results are remarkably close to each other - characteristics always show the same trend and the comparison of some specified values obtained experimentally and theoretically is roughly equal. This fact showed the considerable possibilities of using our experiment as a good measuring method employed to determine parameters that describe liquid crystal materials properties. For example, knowing an electric anisotropy $\Delta \varepsilon$ we can evaluate the elastic constants by measurement of the threshold field of the Frederiks transition.

The fundamental problem in an effective using of proposed method is to match the theoretical and experimental conditions. Obtainment of ideal results is possible only in case of perfectly ordered layer, properly appointed width of beam in a cell surface, and an assurance of ideal geometry of the system. It is also necessary to remark that experimental values - for example: temperature, beam diameter, cell location, and others might be changed during an experiment. On the other hand, the cells are never ideal, so their initial orientation may be different from assumed one.

Nonlinear phenomena caused by molecular reorientation due to influence of external fields seem to be very useful in applications to optoelectronic functional elements. In a short time, we can expect an increase in number of the optical systems and elements, in which the unique properties of the shown reorientational nonlinear mechanism will be used.

\section{Acknowledgments}

Authors would like to express their gratitude to Dr Marek Sierakowski for technical support. This work was partially supported by the Committee for Scientific Research under the grant No. 8 T11D 01915. 


\section{References}

[1] I.C. Khoo, S.T. Wu, Optics and Nonlinear Optics of Liquid Crystals, World Sci., Singapore 1993.

[2] N.V. Tabiryan, A.V. Sukhov, B.Ya. Zeldovich, Mol. Cryst. Liq. Cryst. 136, 1 (1986).

[3] I.C. Khoo, T.H. Liu, P.Y. Yan, J. Opt. Soc. Am. B 4, 115 (1987).

[4] W.K. Bajdecki, M.A. Karpierz, Acta Phys. Pol. A 95, 793 (1999).

[5] L.B. Au, L. Solymar, C. Dettmann, J. Schwartz, Physica A 174, 94 (1991).

[6] L. Calero, W.K. Bajdecki, R. Meucci, Opt. Commun. 168, 201 (1999).

[7] W.K. Bajdecki, L. Calero, R. Meucci, Opt. Commun. 176, 473 (2000). 